\title{
Effects of Mogrosides on High-Fat-Diet-Induced Obesity and Nonalcoholic Fatty Liver Disease in Mice
}

\author{
Xiaobing Zhang ${ }^{1,2}$, Yunfei Song ${ }^{3}$, Yipei Ding ${ }^{1,2}$, Wei Wang ${ }^{4,5}$, Ling Liao ${ }^{4,5}$, Jin Zhong ${ }^{4,5}$, \\ Pengbo Sun ${ }^{4,5}$, Fan Lei ${ }^{6}$, Yaou Zhang ${ }^{2,4,5}$ and Weidong Xie ${ }^{2,4,5, *}$ \\ 1 Department of Chemistry, Tsinghua University, Beijing 100084, China; zxb17@mails.tsinghua.edu.cn (X.Z.); \\ 15139915181@163.com (Y.D.) \\ 2 State Key Laboratory of Chemical Oncogenomics, Graduate School at Shenzhen, Tsinghua University, \\ Shenzhen, China; zhangyo@sz.tsinghua.edu.cn \\ 3 Guilin Layn Natural Ingredients Corp., Guilin 541100, China; song.yunfei@layn.com.cn \\ 4 Open FIESTA Center, Tsinghua University, Shenzhen 518055, China; kappa33wei@live.com (W.W.) \\ 15602927350@163.com (L.L.); zhongjin1996@163.com (J.Z.); pb_sun@foxmail.com (P.S.) \\ 5 Key Lab in Healthy Science and Technology, Division of Life Science, Graduate School at Shenzhen; \\ Tsinghua University, Shenzhen 518055, China \\ 6 Laboratory of Pharmaceutical Science, School of Life Science, School of Medicine, Tsinghua University, \\ Beijing 100084, China; lifn@163.com \\ * Correspondence: xiewd@sz.tsinghua.edu.cn; Tel.: +86-755-2603-6086
}

Received: 12 July 2018; Accepted: 28 July 2018; Published: 29 July 2018

\begin{abstract}
Obesity and nonalcoholic fatty liver disease (NAFLD) are highly prevalent and cause numerous metabolic diseases. However, drugs for the prevention and treatment of obesity and NAFLD remain unavailable. In this study, we investigated the effects of mogrosides (luo han guo, LH) in Siraitia grosvenorii saponins on high-fat-diet-induced obesity and NAFLD in mice. We found that compared with the negative control, LH reduced body and liver weight. LH also decreased fat accumulation and increased AMP-activated protein kinase (AMPK) phosphorylation (pAMPK) levels in mouse livers. We also found that high-purity mogroside $\mathrm{V}$ upregulated pAMPK expression in HepG2 cells. In addition, high-purity mogroside $V$ inhibited reactive oxygen species production and upregulated sequestosome-1 (SQSTM1, p62) expression in THP-1 cells. These results suggest that LH may affect obesity and NAFLD by enhancing fat metabolism and antioxidative defenses. Mogroside V may be a main component of LH. However, the exact molecular mechanisms and active components responsible for the inhibitory effects of LH on obesity and NAFLD require further investigation.
\end{abstract}

Keywords: Siraitia grosvenorii; mogrosides; obesity; nonalcoholic fatty liver disease; AMPK; p62

\section{Introduction}

Obesity is defined as abnormal or excessive fat accumulation or a body mass index $\geq 30 \mathrm{~kg} / \mathrm{m} \mathrm{[1]}$. Obesity rates in low- and middle-income countries are increasing. One-third of the approximately 2 billion individuals who are overweight are considered obese [2]. Obesity exerts severe health effects, degrades life quality, and imposes a massive social burden. However, ideal drugs for the prevention and treatment of obesity are currently unavailable.

Nonalcoholic fatty liver disease (NAFLD) is the most common chronic liver disease. It may progress through the stages of simple bland steatosis, nonalcoholic steatohepatitis, hepatic fibrosis, and cirrhosis to hepatocellular carcinoma [3]. NAFLD is highly prevalent among overweight and obese individuals [4], and a definitive pharmacotherapy for NAFLD remains nonexistent.

Individuals with obesity and NAFLD often consume a high proportion of fat $[5,6]$. The consumption of carbohydrates and sugar-sweetened beverages has been linked with adult 
and childhood obesity and NAFLD [7,8]. Sufficient scientific evidence suggests that decreasing the consumption of sugar-sweetened beverages will reduce the prevalence of obesity and obesity-related diseases [9]. Therefore, there is a large demand for sugar substitutes with high sweetness and low caloric content, especially among obese individuals.

Mogrosides (luo han guo, LH) are the main active components of Siraitia grosvenorii saponins, which are 200-350 times sweeter than sucrose [10]. LH has been extensively used in beverages owing to its high sweetness and nontoxicity. However, whether LH affects obesity and NAFLD remains to be determined.

In this study, we investigated the effects of LH on high-fat-diet-induced obesity and NAFLD in mice. Moreover, we preliminarily analyzed the active mechanisms underlying the effects of mogrosides.

\section{Results}

\subsection{Effects of LH on Biochemical Parameters}

After 4 weeks on a high-fat diet, mice in the high-fat control group had higher body weight than mice in the normal group (Figure 1A). However, the body weight of mice in the treatment groups treated with LH at dosages of 400 and $800 \mathrm{mg} / \mathrm{kg} /$ day was lower than that of mice in the high-fat control group. The body weight of mice in the $60 \mathrm{mg} / \mathrm{kg} /$ day Orlistat (OL) group was lower than that of mice in the high-fat control group.

A

C
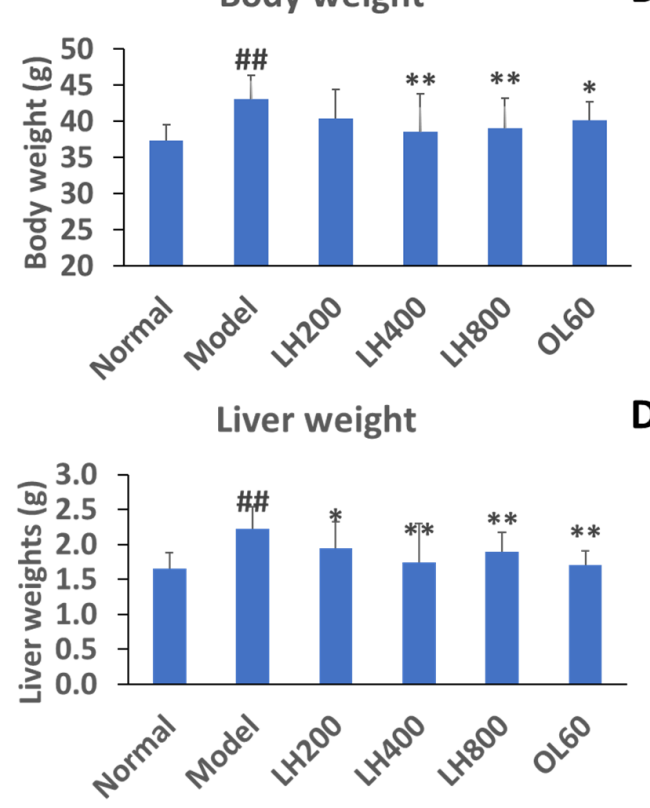

B

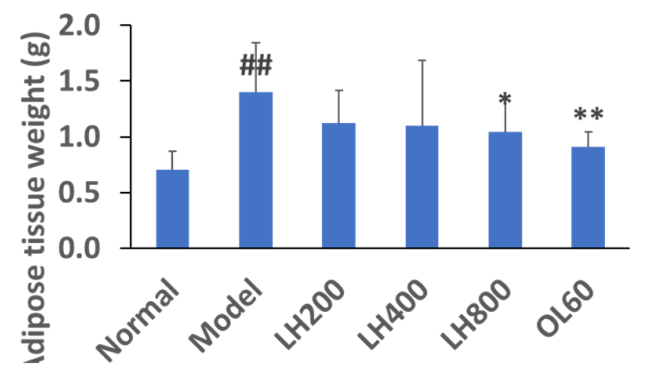

D

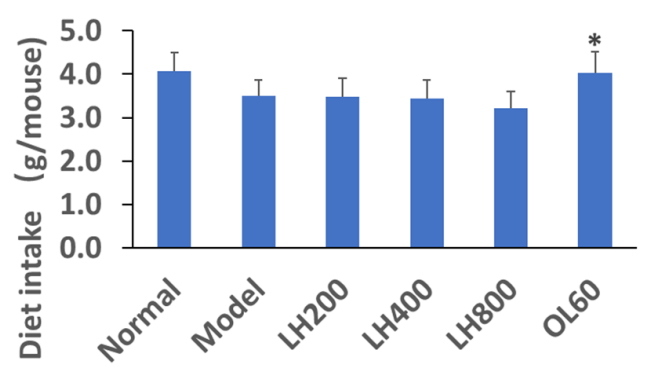

Figure 1. Effects of mogrosides (luo han guo, LH) on (A) body, (B) abdominal adipose, and (C) liver tissue weight and (D) diet intake of mice. Data are presented mean $\pm \mathrm{SD}, \sharp \sharp$ $P<0.01$ vs normal group; ${ }^{*} P<0.05$ and ${ }^{* *} P<0.01$ vs. high-fat control group, $\mathrm{n}=10$. Normal, normal group; model, high-fat control group. LH200, LH400, and LH800: groups orally treated with LH at dosages of 200, 400, and $800 \mathrm{mg} / \mathrm{kg} /$ day, respectively; OL60: group orally treates with Orlistat (OL) at $60 \mathrm{mg} / \mathrm{kg} /$ day.

The weight of abdominal adipose tissues of mice in the high-fat control group was higher than that of mice in the normal group (Figure 1B). The weight of abdominal adipose tissues of mice in the $800 \mathrm{mg} / \mathrm{kg} /$ day LH group and $60 \mathrm{mg} / \mathrm{kg} /$ day OL group was lower than that of mice in the high-fat control group. 
The liver weight of mice in the high-fat control group was significantly higher than that of mice in the normal group (Figure 1C); mice in the 200, 400, and $800 \mathrm{mg} / \mathrm{kg} /$ day LH groups; and mice in the $60 \mathrm{mg} / \mathrm{kg} /$ day OL group.

The food intake of the LH groups and the high-fat control group at $24 \mathrm{~h}$ after LH treatment did not significantly differ (Figure 1D). However, the food intake of the OL group at $24 \mathrm{~h}$ after OL treatment was significantly higher than that of the high-fat control group.

\subsection{Effects of LH on Blood Glucose, Lipid, and Fecal TG Levels}

After 4 weeks on a high-fat diet, the fasting blood glucose and cholesterol levels of the high-fat control group were higher than those of the normal group; however, blood triglyceride (TG) levels of the high-fat control group did not change (Figure 2A-C). Moreover, the blood glucose and lipid levels of mice did not change after 4 weeks of treatment with orally administered OL. Compared with the normal group, the fecal TG content of the high-fat control did not increase. Fecal TG content was unaffected by LH treatment but increased under OL treatment.

A

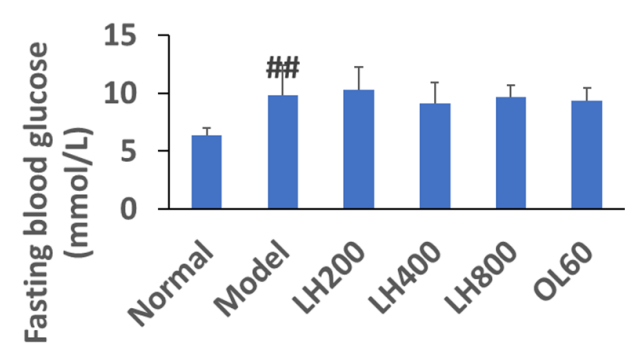

C

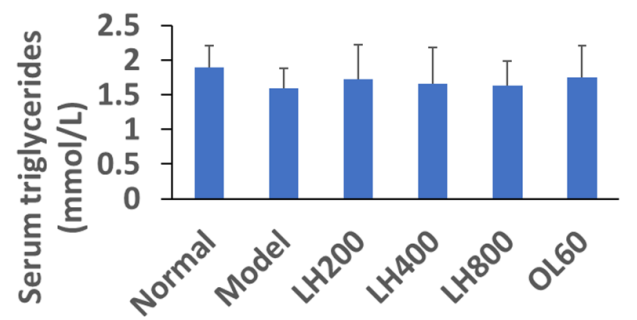

B
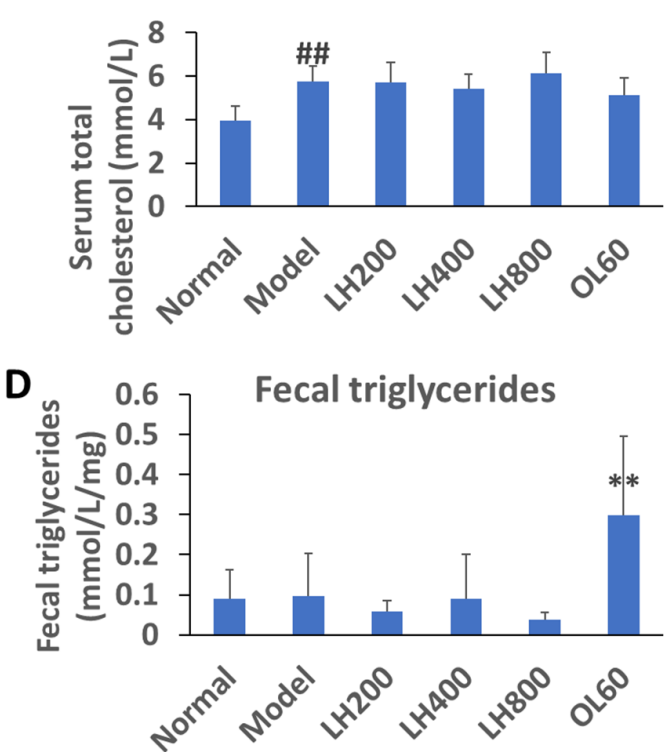

Figure 2. Effects of LH on (A) blood glucose, (B, C) lipid, and (D) fecal triglyceride (TG) levels of mice. Data are presented as mean $\pm \mathrm{SD},{ }^{\# \#} P<0.01$ vs normal group; ${ }^{* *} P<0.01$ vs high-fat control group, $\mathrm{n}=10$. Normal, normal group; model, high-fat control group. LH200, LH400, and LH800: groups orally treated with $\mathrm{LH}$ at dosages of 200,400 , and $800 \mathrm{mg} / \mathrm{kg}$ /day, respectively; OL60: group orally treated with OL at $60 \mathrm{mg} / \mathrm{kg} /$ day.

\subsection{Effects of LH on Abdominal Adipose Tissue Size, Liver Morphology, and Pathology}

After 4 weeks on a high-fat diet, the size of abdominal adipocytes of the high-fat control group increased compared with that of the normal group, as inferred from pathological slices. Given that treatment with $800 \mathrm{mg} / \mathrm{kg}$ /day of LH decreased abdominal adipose tissue, this dosage group was selected for further investigation. Compared with the size of abdominal adipocytes from the high-fat control group, the size from the $800 \mathrm{mg} / \mathrm{kg} /$ day LH group and $60 \mathrm{mg} / \mathrm{kg}$ OL group significantly decreased (Figure 3). 

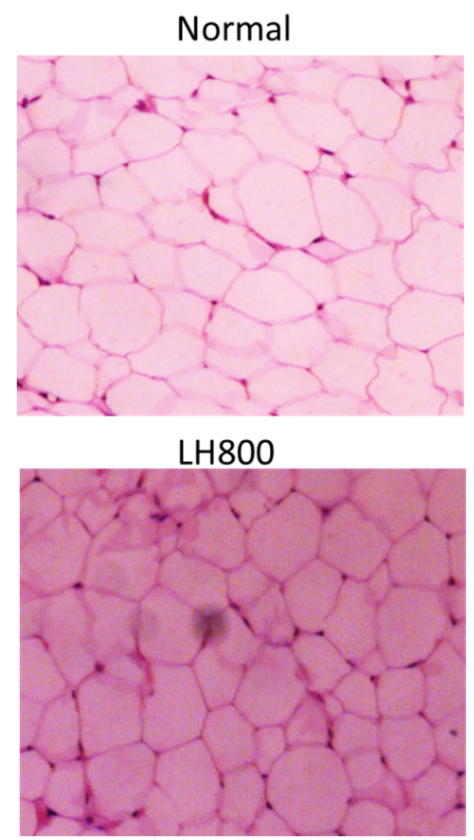

Model

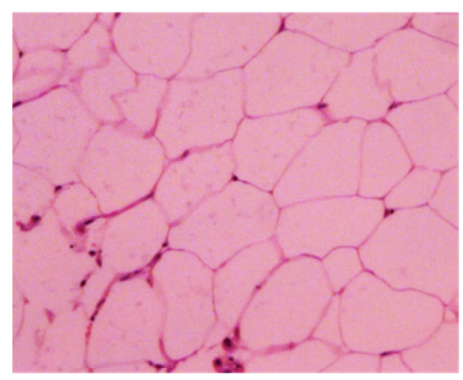

OL60

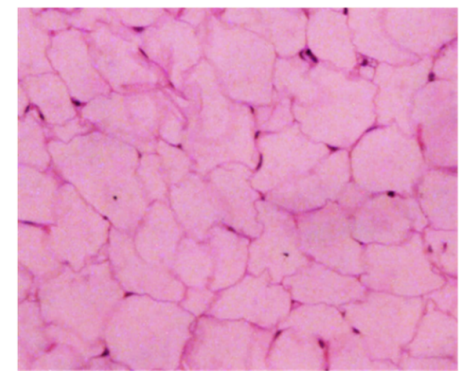

Figure 3. Effects of LH on the size of abdominal adipocytes of mice. Hematoxylin and eosin (H\&E) staining (100× magnification). Normal, normal group; model, high-fat control group. LH800: group orally treated with $\mathrm{LH}$ at dosage of $800 \mathrm{mg} / \mathrm{kg} /$ day; OL60: group orally treated with OL at $60 \mathrm{mg} / \mathrm{kg} /$ day $(\mathrm{n}=10)$.

Meanwhile, the liver color of the high-fat control group appeared light yellow and that of the normal group was dark red. The liver color of groups treated with different dosages of LH and OL changed from light yellow to dark red (Figure 4).

Normal

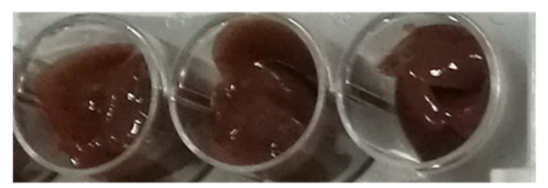

Model

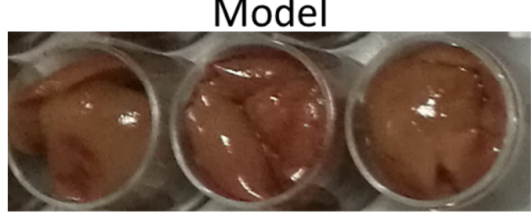

LH2O0

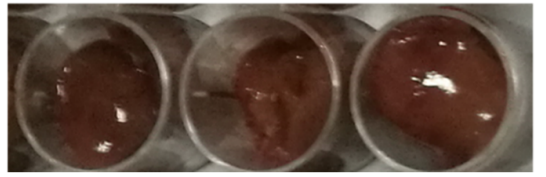

LH400

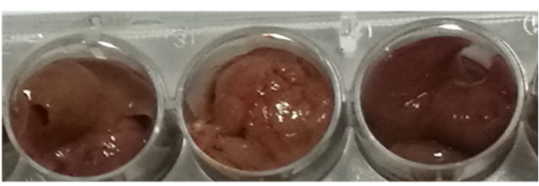

LH800

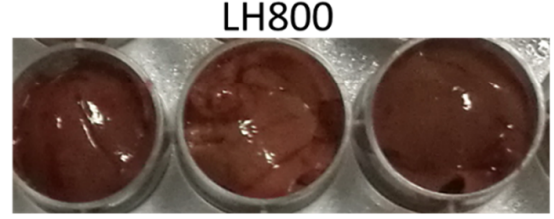

OL60

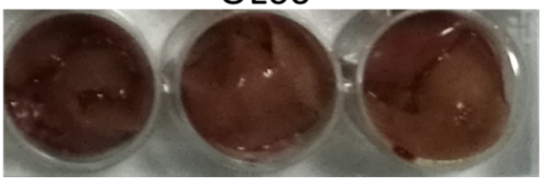

Figure 4. Effects of LH on the color of mouse livers. Normal, normal group; model, high-fat control group. LH200, LH400, and LH800: groups orally treated with LH at dosages of 200, 400, and $800 \mathrm{mg} / \mathrm{kg} /$ day, respectively; OL60: group orally treated with OL at $60 \mathrm{mg} / \mathrm{kg} /$ day $(\mathrm{n}=10)$.

Furthermore, histopathology analysis revealed that mice in the high-fat control group showed remarkable liver steatosis (Figure 5). However, fat accumulation decreased in mice in groups that were treated with different dosages of LH and OL. 


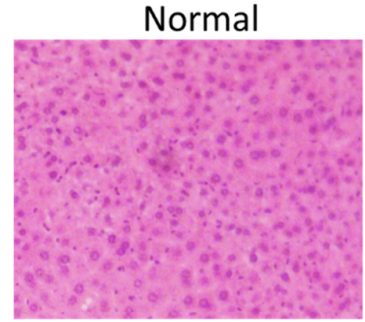

LH400

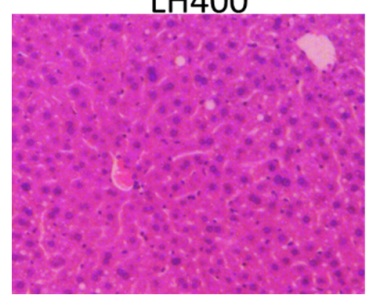

Model

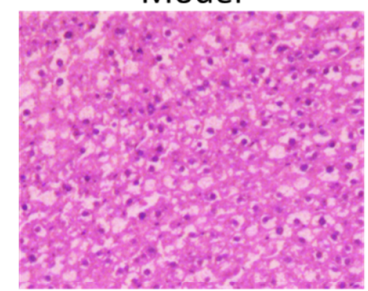

LH800

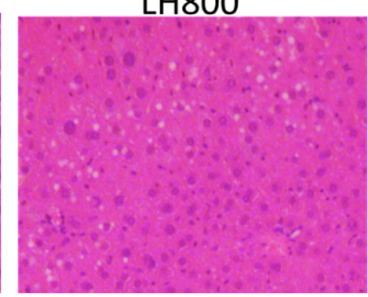

LH200

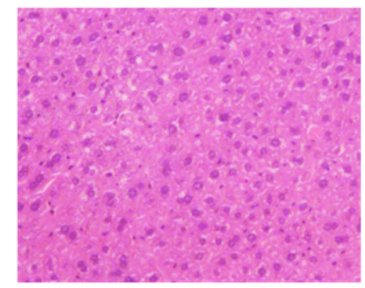

OL60

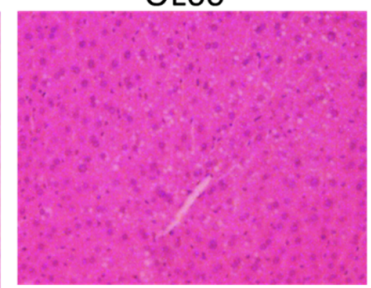

Figure 5. Effects of LH on the liver pathology of mice. H\&E staining (100× magnification). Normal, normal group; model, high-fat control group. LH200, LH400, and LH800: groups orally treated with $\mathrm{LH}$ at dosages of 200,400, and $800 \mathrm{mg} / \mathrm{kg} /$ day, respectively; OL60: group orally treated with OL at $60 \mathrm{mg} / \mathrm{kg} /$ day $(\mathrm{n}=10)$.

\subsection{Effects of LH on Serum ALT and AST Activity}

Serum alanine aminotransferase (ALT) and aspartate aminotransferase (AST) activity increased in mice fed high-glucose and high-fat diets (Figure 6A,B). However, this increase was significantly attenuated by treatment with different dosages of LH and OL.
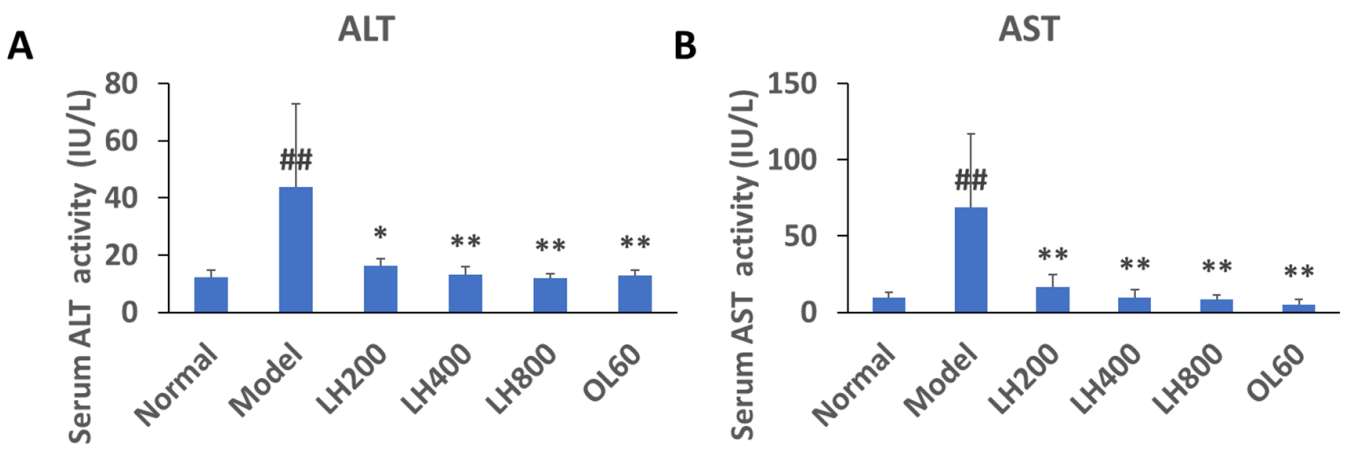

Figure 6. Effects of LH on (A) serum alanine aminotransferase (ALT) and (B) aspartate aminotransferase (AST) activity in mice. Data are presented as mean $\pm \mathrm{SD},{ }^{\# \#} P<0.01$ vs normal group; ${ }^{*} P<0.05$ and ** $P<0.01$ vs. high-fat control group, $\mathrm{n}=10$. Normal, normal group; model, high-fat control group. LH200, LH400, and LH800: groups orally treated with LH at dosages of 200, 400, and $800 \mathrm{mg} / \mathrm{kg} / \mathrm{day}$, respectively; OL60: group orally treated with OL at $60 \mathrm{mg} / \mathrm{kg} /$ day.

\subsection{Effects of LH on Liver AMPK Phosphorylation}

pAMPK expression was upregulated in the livers of mice that received $200-800 \mathrm{mg} / \mathrm{kg} \mathrm{LH}$ orally (Figure 7A). Meanwhile, pAMPK expression levels increased in HepG2 cells incubated with $20-80 \mu \mathrm{g} / \mathrm{mL}$ high pure mogroside $\mathrm{V}$, one of the main components of LH (Figure 7B). 


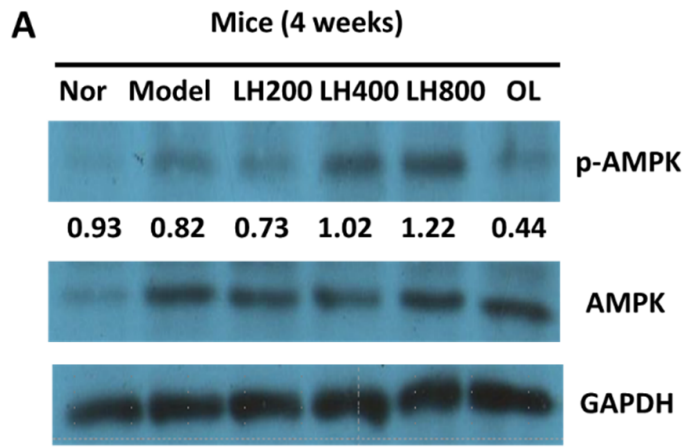

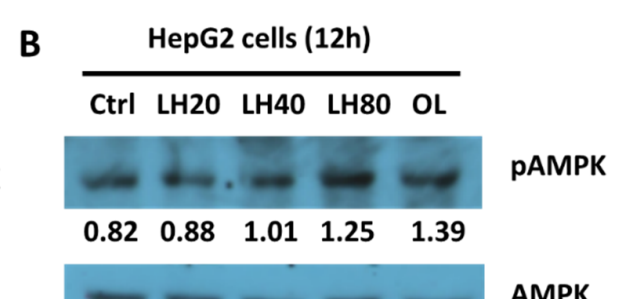
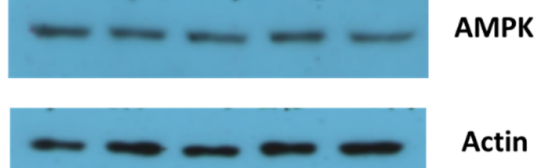

Figure 7. Effects of LH on pAMPK expression in (A) mouse livers and (B) HepG2 cells. Nor, normal group; model, high-fat control group; Ctrl, control. LH200, LH400, and LH800: groups orally treated with LH at dosages of 200,400, and $800 \mathrm{mg} / \mathrm{kg}$ /day, respectively; OL60: group orally treated with OL at $60 \mathrm{mg} / \mathrm{kg} /$ day $(\mathrm{n}=10)$. LH20, LH40, and LH80: LH (high pure mogroside $\mathrm{V}$ ) at final concentrations of 20,40 , and $80 \mu \mathrm{g} / \mathrm{mL}$, respectively $(\mathrm{n}=3)$.

\subsection{Effects of LH on Antioxidative Defenses in THP-1 Cells}

Reactive oxygen species (ROS) production increased in lipopolysaccharide (LPS)- and phorbol-12-myristate-13-acetate (PMA)-induced THP-1 cells and was inhibited by mogroside V treatment (Figure 8A,B). Hence, p62 expression in LPS- and PMA-induced THP-1 cells was assayed (Figure 8C). Compared with the control treatment, mogroside $\mathrm{V}$ treatment increased p62 expression. The above results prove that mogroside $\mathrm{V}$ can promote the activation of antioxidative defenses.

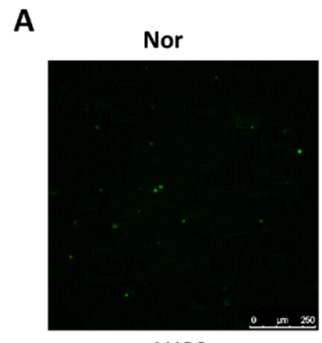

LH20

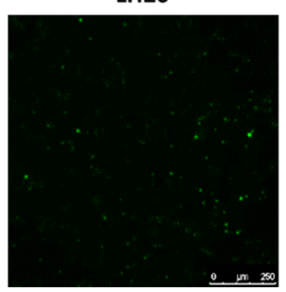

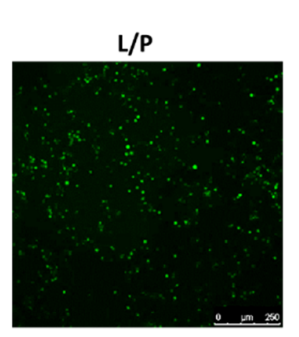

LH40

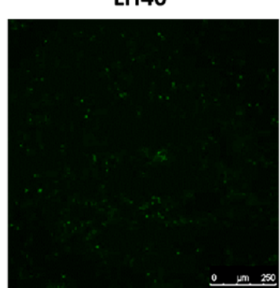

B

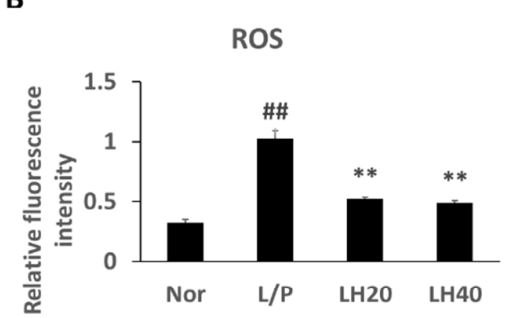

C

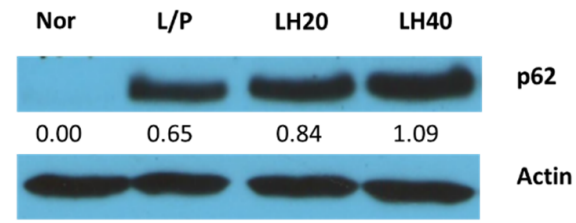

Figure 8. (A,B) Effects of LH on reactive oxygen species (ROS) and (C) p62 levels in THP-1 cells. Nor, normal control; L/P, LPS- and PMA-induced control group. LH20 and LH40: LPS- and PMA-induced groups simultaneously treated with LH (high pure mogroside V) at final concentrations of 20 and $40 \mu \mathrm{g} / \mathrm{mL}$, respectively. Data are presented as mean $\pm \mathrm{SD},{ }^{\#} P<0.01$ vs. normal control group; ** $P<0.01$ vs LPS- and PMA-induced control group $(n=3)$.

\section{Discussion}

An important tactic in the prevention or treatment of weight gain and obesity is usually inhibiting dietary intake and consumption. However, many drugs for curing obesity have been withdrawn because of serious side effects [11]. OL as a positive drug reduced gut fat absorption by inhibiting the activity of pancreatic lipase, but was still little used [12]. The results of this study provide preliminary evidence that LH inhibits obesity and fatty liver induced by a high-fat diet. The antiobesity effect of 
LH does not appear to be associated with decreased dietary intake or decreased fat absorption by the gut. Considering that we tested the antiobesity effect of LH in mice only within 1 month, a longer term of effect or safety evaluation needs to be further investigated in the future.

LH may protect against the development of NAFLD by inhibiting fat droplet formation in the liver. NAFLD is associated with increased liver size, and hepatomegaly may be associated with hepatocyte enlargement caused by lipid accumulation [13]. Elevated serum AST and ALT activity indicates that liver cells may have been subjected to inflammatory damage [14]. Increased fat accumulation, inflammation, and oxidative stress in the liver may contribute to the development of NAFLD [15]. Actually, NAFLD is a complicated disease. Hormone, growth factor, and adipokine imbalance also plays an important role in the development of NAFLD [16]. AMPK is an important factor in the development of NAFLD, because its phosphorylation is responsible for the activation of fatty acid oxidation and energy metabolism [17]. Promoting AMPK activity is a viable therapeutic strategy for NAFLD [18]. The present work shows that mogroside treatment upregulates pAMPK expression in mouse livers. This effect was further validated in HepG2 cells. These results suggest that LH promotes fat oxidation metabolism to inhibit the development of NAFLD.

LH exhibited anti-inflammatory effects in LPS-induced mice [19] and inhibited ROS production in LPS- and PMA-induced THP-1 cells. LPS and PMA increased ROS production in monocytes and macrophage cells $[20,21]$. NAFLD is associated with macrophage recruitment and consequent inflammation after adipocyte enlargement [22]. Oxidative stress also plays an important role in the development of NAFLD [23]. Immune cells, instead of liver cells, may contribute to the development of inflammation or oxidative stress in liver tissues. p62 is an important antioxidative defense factor that is also involved in autophagy [24]. In THP-1 cells, LH considerably increased p62 expression, although LPS combined with PMA also compensatively increased p62 expression. These results indicate that the potential effect of LH on oxidative stress or inflammation in livers may be associated with the increased capacity for antioxidative defense through the upregulation of p62 expression.

LH inhibits obesity and NAFLD. The action mechanisms underlying this inhibitory effect may be associated with the promotion of AMPK phosphorylation and enhancement of antioxidative defenses through the upregulation of p62 expression. Mogroside V may be an active component of LH. LH does not affect blood glucose and lipid levels despite its sweet taste. Hence, it might be used as a natural alternative sweetener in food products for patients with NAFLD, obesity, diabetes, or hyperlipidemia. Nevertheless, the exact molecular mechanisms underlying the antiobesity and anti-NAFLD action of LH require further investigation.

\section{Materials and Methods}

\subsection{Chemicals and Reagents}

LH (approximately 46\% mogroside V, 6.8\% 11-oxo-mogroside V, 3.2\% mogroside IV, $g / g$ ) was purchased from Guilin Layn Natural Ingredients Corp. (Guilin, China). HPLC of LH is shown in Figure 9. High-purity mogroside V (98\%) was obtained from Dasf Biotechnology Co., Ltd. (Nanjing, China). OL was purchased from Zhejiang Hisun Pharmaceutical Co., Ltd. (Taizhou, China). Glucose, total cholesterol (TC), and TG kits were purchased from BioSino Bio-Technology \& Science Inc. (Beijing, China). ALT and AST kits were obtained from Nanjing Jiancheng Institute of Biotechnology (Nanjing, China). Bradford Protein Assay Kit was purchased from Beyotime Research Institute of Biotechnology (Shanghai, China). 


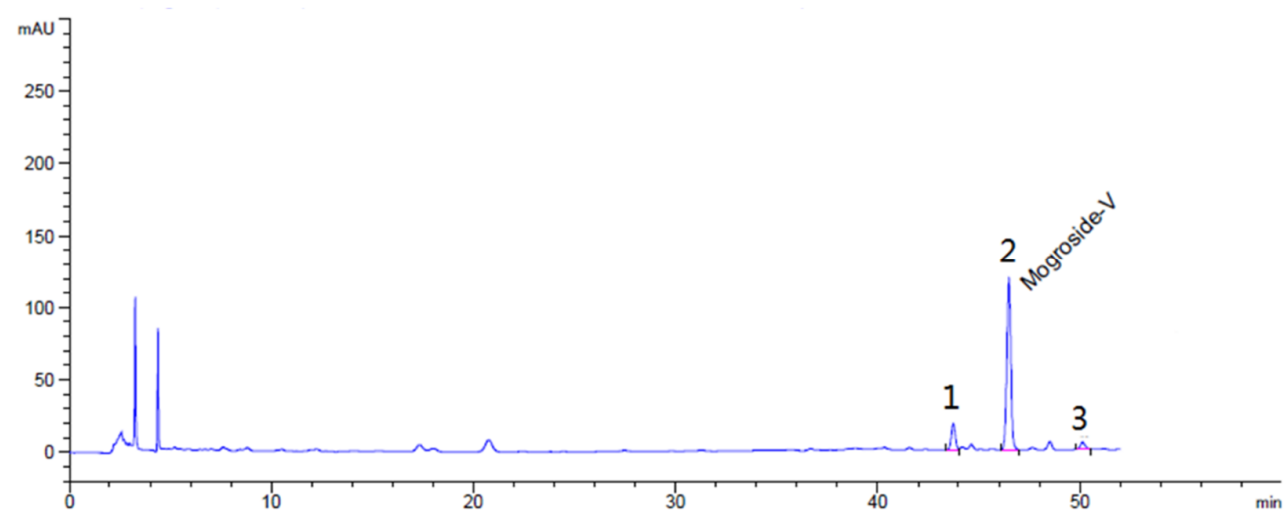

Figure 9. HPLC of Siraitia grosvenorii LH. Peak 1, 11-oxo-mogroside V, 6.8\% $(\mathrm{g} / \mathrm{g})$; peak 2, mogroside $\mathrm{V}, 46 \%(\mathrm{~g} / \mathrm{g})$; peak 3 , mogroside IV, $3.2 \% \mathrm{~g} / \mathrm{g}$.

\subsection{High-Fat-Diet-Induced Mice}

Sixty 4-week-old male NIH mice weighing 18-22 g were purchased from Guangdong Medical Laboratory Animal Center (Guangzhou, China). The animals were maintained in a room at a temperature of $20 \pm 2{ }^{\circ} \mathrm{C}$ and humidity of $60 \% \pm 5 \%$ under a $12 \mathrm{~h}$ dark/light cycle. All experiments were performed strictly in accordance with the recommendations of the Guide for the Care and Use of Laboratory Animals of the Institutional Animal Care and Use Committee of Tsinghua University (13_LQF3). The mice were randomly divided into 6 groups ( $\mathrm{n}=10$ per group): normal, high-fat control, low-dose LH, medium-dose LH, high-dose $\mathrm{LH}$, and positive. The normal group received a normal chow diet (weight ratio: $4 \%$ fat, $65 \%$ carbohydrates, $20 \%$ protein, $g / g$ [25]; $10 \%$ of total calories from fat; $3.58 \mathrm{Kcal} / \mathrm{g}$ diet; Guangdong Medical Animal Center, Foshan, China), and the remaining groups received high-fat diets (weight ratio: $20 \%$ fat, $50 \%$ carbohydrates, $21 \%$ protein, $g / g ; 41 \%$ of total calories from fat; $4.7 \mathrm{Kcal} / \mathrm{g}$ diet; H10141; Beijing HFK Bioscience Co., Ltd., Beijing, China). LH was orally administered to the $3 \mathrm{LH}$ groups at dosages of 200,400 , and $800 \mathrm{mg} / \mathrm{kg} /$ day. OL, the positive control, was orally administered to the positive control group at a dosage of $60 \mathrm{mg} / \mathrm{kg} /$ day. LH and OL were freshly prepared by dissolution in deionized water. Equal volumes of deionized water were fed to the normal and high-fat control groups. The food and water intake of the animals within $24 \mathrm{~h}$ of drug administration was observed twice a week. In the third week of treatment, fecal samples were collected and stored at $-80^{\circ} \mathrm{C}$ until assayed. After 4 weeks, the mice were fasted from 9:00 a.m. to 3:00 p.m. and anesthetized by intraperitoneal injection with $10 \%$ urethane solution $(\mathrm{g} / \mathrm{mL}$, dissolved in normal saline; Sangon Biotech, Shanghai, China) at a dosage of $10 \mathrm{~mL} / \mathrm{kg}$. Blood samples were collected from the orbital plexus. Serum samples were obtained from the collected blood samples through $5 \mathrm{~min}$ of centrifugation $\left(5000 \mathrm{rpm}, 4{ }^{\circ} \mathrm{C}\right.$ ) and stored at $-20^{\circ} \mathrm{C}$ until assayed. Liver and abdominal adipose tissues were rapidly removed and weighed. Portions of the liver and abdominal adipose tissue were stored in $4 \%$ paraformaldehyde solution for conventional hematoxylin and eosin (H\&E) staining and slice analysis, and the remaining tissues were stored at $-80{ }^{\circ} \mathrm{C}$ for further biochemical analysis.

\subsection{Biochemical Analysis}

Blood glucose, TG, TC, AST, and ALT levels were assayed in accordance with the protocols provided with commercial kits. Feces (approximately $100 \mathrm{mg}$ ) was collected from each mouse, placed in $1.5 \mathrm{~mL}$ Eppendorf tubes, and dried in an oven at $60^{\circ} \mathrm{C}$ for $30 \mathrm{~min}$. Then, the dried feces was soaked for $30 \mathrm{~min}$ in $200 \mu \mathrm{L}$ of PBS. The feces was homogenized in $800 \mu \mathrm{L}$ of chloroform-methanol mixture $(2: 1, v / v)$. The homogenates were maintained for $5 \mathrm{~min}$ at room temperature and then centrifuged for $5 \mathrm{~min}$ at $12,000 \times \mathrm{g}$ and $4{ }^{\circ} \mathrm{C}$. Subsequently, $50 \mu \mathrm{L}$ of the supernatants was transferred into Eppendorf tubes and dried in an oven for $1 \mathrm{~h}$ at $40^{\circ} \mathrm{C}$. The supernatants were incubated for 30-60 min in $250 \mu \mathrm{L}$ of the working solution included with the TG kit. Finally, TG levels were quantified. 


\subsection{Cell Culture}

HepG2 and THP-1 cells were provided by the Cell Resource Center of the Shanghai Institute for Biological Sciences, Chinese Academy of Sciences, Shanghai, China. Both cell lines were cultured in Dulbecco's Modified Eagle's Medium (high glucose, Gibco ${ }^{\circledR}$, Thermo Fisher Scientific, Waltham, MA, USA) supplemented with 10\% fetal bovine serum (FBS; Premium, Pan Biotech, Aidenbach, Germany) and $1 \%$ pen-strep antibiotics (Gibco ${ }^{\mathrm{TM}}$, Thermo Fisher Scientific, USA). Then, the cell lines were incubated in a humidified atmosphere at $37^{\circ} \mathrm{C}$ with $5 \% \mathrm{CO}_{2}$. The cells were seeded into 6-well plates at a density of $2.5 \times 10^{5}$ cells per well.

We assayed the cytotoxicity of high-purity mogroside $\mathrm{V}$, one of the main components of LH, in HepG2 and THP-1 cells. Mogroside V at final concentrations of not more than $100 \mu \mathrm{g} / \mathrm{mL}$ within $48 \mathrm{~h}$ of incubation did not show any significant cytotoxic response on (3-(4,5-dimethyl-2-thiazolyl)-2,5-diphenyl-2- $H$-tetrazolium bromide (MTT) assay (MTT cell proliferation and cytotoxicity assay kit, Nanjing Jiancheng Bioengineering Institute, Nanjing, China). HepG2 cells were used to investigate the effects of high-purity mogroside V on pAMPK expression in mouse livers. After $12 \mathrm{~h}$ of attachment, HepG2 cells were incubated for $12 \mathrm{~h}$ with mogroside $\mathrm{V}$ at final concentrations of 20-80 $\mu \mathrm{g} / \mathrm{mL}$, and cell lysates were collected for Western blot analysis.

The effects of high-purity mogroside $\mathrm{V}$ treatment on reactive oxygen species (ROS) production were investigated in THP-1 cells. Cells were incubated for $12 \mathrm{~h}$ with mogroside $\mathrm{V}$ at final concentrations of 20 and $40 \mu \mathrm{g} / \mathrm{mL}$. Cell lysates were collected for further Western blot analysis.

\subsection{ROS Assay}

The ROS assay was conducted in accordance with previous methods [20]. In this assay, 2,7-dichlorodi-hydrofluorescein diacetate (DCFH-DA) fluorescence probes were transported into cells and then hydrolyzed into DCFH. Intracellular active oxygen binds to DCFH and causes it to emit fluorescence that can be detected by fluorescence microscopy (excitation wavelength $485 \mathrm{~nm}$; emission wavelength $525 \mathrm{~nm})$. Here, THP-1 cells $\left(5 \times 10^{4}\right.$ per well) were seeded into 24-well plates and incubated for $12 \mathrm{~h}$. Subsequently, mogroside V was added at final concentrations of 20-40 $\mu \mathrm{g} / \mathrm{mL}$. After $30 \mathrm{~min}$ of incubation, the cells were incubated for 6-24 h with LPS $(2 \mu \mathrm{g} / \mathrm{mL}$ final concentration) and PMA (200 ng/mL final concentration). Subsequently, the medium in each plate was replaced with fresh FBS-free medium containing DCFH-DA (10 mM final concentration), and the plates were incubated for $20 \mathrm{~min}$. Then, the cells were washed thrice with fresh FBS-free medium and immediately observed under fluorescence microscopy at 200× magnification (Leica DMI6000B, Heidelberg, Germany). Six random areas with the same size were selected from the captured images of each sample. The gray density of fluorescence intensity in each area was calculated using ImageJ software (version 1.48, National Institutes of Health, Bethesda, MD, USA), and the average value of each sample was obtained from 3 biological replicates for further statistical analysis. The average gray density values of fluorescence intensity from the negative controls treated with LPS and PMA were defined as 1 . All other values were normalized on the basis of this value.

\subsection{Western Blot Analysis}

Approximately $100 \mathrm{mg}$ of liver tissue from each mouse was dissolved in $1 \mathrm{~mL}$ of cell lysate $(50 \mathrm{mM}$ Tris-HCl, 4 M urea, and 1\% Triton X-100, pH 8.0). Western blot analysis was conducted with liver homogenates (approximately $50 \mu \mathrm{g}$ of the protein samples). Protein levels were quantified by using the Bradford Protein Assay Kit. Target proteins were separated via SDS-PAGE electrophoresis and then transferred from gels to PVDF membranes under $220 \mathrm{~mA}$ for $1.5 \mathrm{~h}$. After blocking with $5 \%$ bovine serum albumin for $1.5 \mathrm{~h}$, the membranes were incubated overnight with primary antibodies at $4{ }^{\circ} \mathrm{C}$. The primary antibodies included mouse polyclonal $\beta$-actin antibody (1:5000, A1978; Sigma-Aldrich Co., St. Louis, MO, USA) or rabbit polyclonal antibodies against GAPDH (1:2000, Cat. No. ABS118936A; Absin Bioscience Inc., Beijing, China), rabbit antibodies against AMPK and phospho-AMPK $\alpha$ (Thr172) 
(1:1000, Cat. No. 2532 and 2535, respectively; Cell Signaling Technology, Inc., Danvers, MA, USA), and p62 (1:2000, PM045; Medical \& Biological Laboratories Co., Ltd., Nagoya, Japan). After incubation, the membranes were washed 4 times with Tris-buffered saline with Tween 20 (TBST) for 10 min per wash. Next, the membranes were incubated for $1.5 \mathrm{~h}$ with secondary antibodies, including horseradish-peroxidase-labeled goat anti-rabbit lgG H\&L (1:5000, Cat. No. 074-1506; SeraCare/KPL Company, Milford, MA, USA), at room temperature. After incubation, the membranes were washed 5 times with TBST for 10 min per wash. The expression levels of target proteins were detected by using Pierce $^{\mathrm{TM}}$ ECL Western Blotting Substrate (Cat. No. 32106, Thermo Fisher Scientific, USA). Protein levels were normalized to GAPDH or actin levels, and protein quantities were analyzed on the basis of relative gray density values by using ImageJ software (version 1.48, National Institutes of Health, Bethesda, MD, USA).

\subsection{Statistical Analysis}

Data are expressed as mean \pm SD. Statistical significance was evaluated through one-way ANOVA and set as $P<0.05$. When appropriate, Newman-Keuls comparison was used to determine the source of significant differences.

Author Contributions: Formal analysis, X.Z., F.L. and W.X.; investigation, X.Z., Y.D., W.W., L.L., J.Z., and P.S.; project administration, Y.Z.; supervision, Y.S. and W.X.; writing - original draft, X.Z. and W.X.; writing-review and editing, W.X.

Funding: This research was funded by the National Natural Science Foundation of China (Grant No. 81373460) and the Shenzhen Science and Technology Innovation Committee (JCYJ20170307152357168 and GRCK2017042415075836).

Conflicts of Interest: The authors declare no conflict of interest.

\section{References}

1. Secombe, P.; Harley, S.; Chapman, M.; Aromataris, E. Feeding the critically ill obese patient: A systematic review protocol. JBI Database Syst. Rev. Implement. Rep. 2015, 13, 95-109. [CrossRef] [PubMed]

2. Seidell, J.C.; Halberstadt, J. The global burden of obesity and the challenges of prevention. Ann. Nutr. Metab. 2015, 66 (Suppl. 2), 7-12. [CrossRef] [PubMed]

3. Pati, G.K.; Singh, S.P. Nonalcoholic fatty liver disease in south asia. Euroasian J. Hepato-Gastroenterol. 2016, 6, 154-162. [CrossRef] [PubMed]

4. Karim, M.F.; Al-Mahtab, M.; Rahman, S.; Debnath, C.R. Non-alcoholic fatty liver disease (NAFLD)—A Review. Mymensingh Med. J. MMJ 2015, 24, 873-880. [PubMed]

5. Vranesic Bender, D.; Nutrizio, M.; Josic, M.; Ljubas Kelecic, D.; Karas, I.; Premuzic, M.; Domislovic, V.; Rotim, C.; Krznaric, Z. Nutritional status and nutrition quality in patients with non-alcoholic fatty liver disease. Acta Clin. Croatica 2017, 56, 625-634. [CrossRef] [PubMed]

6. Hall, K.D.; Bemis, T.; Brychta, R.; Chen, K.Y.; Courville, A.; Crayner, E.J.; Goodwin, S.; Guo, J.; Howard, L.; Knuth, N.D.; et al. Calorie for calorie, dietary fat restriction results in more body fat loss than carbohydrate restriction in people with obesity. Cell Metab. 2015, 22, 427-436. [CrossRef] [PubMed]

7. White, A.H.; James, S.A.; Paulson, S.W.; Beebe, L.A. Sugar sweetened beverage consumption among adults with children in the home. Front. Nutr. 2018, 5, 34. [CrossRef] [PubMed]

8. Bray, G.A.; Popkin, B.M. Dietary sugar and body weight: Have we reached a crisis in the epidemic of obesity and diabetes?: health be damned! Pour on the sugar. Diabetes Care 2014, 37, 950-956. [CrossRef] [PubMed]

9. Hu, F.B. Resolved: There is sufficient scientific evidence that decreasing sugar-sweetened beverage consumption will reduce the prevalence of obesity and obesity-related diseases. Obes. Rev. 2013, 14, 606-619. [CrossRef] [PubMed]

10. Liu, C.; Dai, L.; Liu, Y.; Dou, D.; Sun, Y.; Ma, L. Pharmacological activities of mogrosides. Future Med. Chem. 2018, 10, 845-850. [CrossRef] [PubMed]

11. Krentz, A.J.; Fujioka, K.; Hompesch, M. Evolution of pharmacological obesity treatments: focus on adverse side-effect profiles. Diabetes Obes. Metab. 2016, 18, 558-570. [CrossRef] [PubMed] 
12. Arch, J.R. Horizons in the pharmacotherapy of obesity. Curr. Obes. Rep. 2015, 4, 451-459. [CrossRef] [PubMed]

13. Liang, W.; Menke, A.L.; Driessen, A.; Koek, G.H.; Lindeman, J.H.; Stoop, R.; Havekes, L.M.; Kleemann, R.; van den Hoek, A.M. Establishment of a general NAFLD scoring system for rodent models and comparison to human liver pathology. PLoS ONE 2014, 9, e115922. [CrossRef] [PubMed]

14. Rej, R. Aminotransferases in disease. Clin. Lab. Med. 1989, 9, 667-687. [PubMed]

15. Engin, A. Non-alcoholic fatty liver disease. Adv. Exp. Med. Biol. 2017, 960, 443-467. [PubMed]

16. Tarantino, G.; Savastano, S.; Colao, A. Hepatic steatosis, low-grade chronic inflammation and hormone/growth factor/adipokine imbalance. World J. Gastroenterol. 2010, 16, 4773-4783. [CrossRef] [PubMed]

17. O'Neill, H.M.; Holloway, G.P.; Steinberg, G.R. AMPK regulation of fatty acid metabolism and mitochondrial biogenesis: implications for obesity. Mol. Cell. Endocrinol. 2013, 366, 135-151. [CrossRef] [PubMed]

18. Smith, B.K.; Marcinko, K.; Desjardins, E.M.; Lally, J.S.; Ford, R.J.; Steinberg, G.R. Treatment of nonalcoholic fatty liver disease: role of AMPK. Am. J. Physiol.-Endocrinol. Metab. 2016, 311, E730-E740. [CrossRef] [PubMed]

19. Shi, D.; Zheng, M.; Wang, Y.; Liu, C.; Chen, S. Protective effects and mechanisms of mogroside V on LPS-induced acute lung injury in mice. Pharm. Biol. 2014, 52, 729-734. [CrossRef] [PubMed]

20. Jiang, X.; Xu, C.; Lei, F.; Liao, M.; Wang, W.; Xu, N.; Zhang, Y.; Xie, W. MiR-30a targets IL- $\alpha$ and regulates islet functions as an inflammation buffer and response factor. Sci. Rep. 2017, 7, 5270. [CrossRef] [PubMed]

21. Xu, C.; Wang, W.; Zhong, J.; Lei, F.; Xu, N.; Zhang, Y.; Xie, W. Canagliflozin exerts anti-inflammatory effects by inhibiting intracellular glucose metabolism and promoting autophagy in immune cells. Biochem. Pharmacol. 2018, 152, 45-59. [CrossRef] [PubMed]

22. Alisi, A.; Carpino, G.; Oliveira, F.L.; Panera, N.; Nobili, V.; Gaudio, E. The role of tissue macrophage-mediated inflammation on NAFLD pathogenesis and its clinical implications. Mediat. Inflamm. 2017, 2017, 8162421. [CrossRef] [PubMed]

23. Klisic, A.; Isakovic, A.; Kocic, G.; Kavaric, N.; Jovanovic, M.; Zvrko, E.; Skerovic, V.; Ninic, A. Relationship between oxidative stress, inflammation and dyslipidemia with fatty liver index in patients with type 2 diabetes mellitus. Exp. Clin. Endocrinol. Diabetes 2018, 126, 371-378. [CrossRef] [PubMed]

24. Wang, L.; Cano, M.; Handa, J.T. p62 provides dual cytoprotection against oxidative stress in the retinal pigment epithelium. Biochim. Biophys. Acta 2014, 1843, 1248-1258. [CrossRef] [PubMed]

25. Xie, W.D.; Wang, H.; Zhang, J.F.; Kung, H.F.; Zhao, Y.N.; Zhang, Y. Proteomic profile of visceral adipose tissues between low-fat diet-fed obesity-resistant and obesity-prone C57BL/6 mice. Mol. Med. Rep. 2010, 3 , 1047-1052. [CrossRef] [PubMed]

Sample Availability: Samples of the compounds (Mogrosides or mogroside V) are available from the authors.

(C) 2018 by the authors. Licensee MDPI, Basel, Switzerland. This article is an open access article distributed under the terms and conditions of the Creative Commons Attribution (CC BY) license (http://creativecommons.org/licenses/by/4.0/). 